\title{
Erklärung zur Unterdrückung von oppositionellen Kräften in Peru
}

Am 25. September 1973 durchsuchte die peruanische Staatssicherheitspolizei die Buchhandlungen Limas und beschlagnahmte die Nr. 4 der kritischen sozialwissenschaftlichen Zeitschrift ,Sociedad y Politica'. Diese Zeitschrift befaßt sich mit der theoretischen Analyse der derzeitigen politökonomischen, sowie der gesellschaftlichen Entwicklung in Peru und fordert den aktiven und autonomen Eingriff des Volkes in diesen Prozeß. Durch ihren Beitrag zum Studium der sozio-ökonomischen Entwicklung und zur Denunzierung der imperialistischen Herrschaft in Lateinameri$\mathrm{ka}$ ist die Zeitschrift weit bekannt. Die Herausgeber werden verfolgt. Der Direktor Anibal Quijano Obregón und der Redakteur Julio Cotler wurden am 6. Oktober 1973, ohne daßs ihnen dafür eine Erklärung gegeben wurde, nach Argentinien ausgewiesen. Welches sind die Hintergründe dieser im Gegensatz zur peruanischen Pressegesetzgebung stehenden Willkürmaßnahmen?

Peru befindet sich seit 1968 unter der Herrschaft einer Militärregierung. Sie löste eine Serie von zivilen und militärischen Regierungen ab, die faktisch das Land durch die Vergabe von Konzessionen an inländische und insbesondere ausländische Kapitalgeber, deren Interessen sie nachher vertraten, verwalte that ten. Beispielsweise stiegen die transferierten Gewinne nordamerikanischer Gesellschaften von 17 Millionen Dollar im Jahre 1958 auf 137 Millionen im Jahre 1966. Sozialpolitik wurde in äußerst geringem Umfang betrieben, sie gipfelte bei der der Militärregierung vorhergehenden zivilen Regierung Belaúnde im Aufruf an die seit Jahrhunde ten ausgebeutete und verelendete Landbevölkerung, sich selber zu helfen, ohne daß die Mechanismen, die zur Verelendung geführt hatten, angegriffen wurden. Die Industrialisierungspolitik war entsprechend dem genannten Konzessionssystem chaotisch und führte in keiner Weise zu einer Absorption der von der verelendeten und stagnierenden Landwirtschaft freigesetzten Arbeitskräfte. Beispielsweise wurden in der Fischmehlindustrie Überkapazitäten angelegt, die Fischgründe weitgehend zerstört, so daß die in einem Jahr gefangenen Fische heute in 50 Tagen verarbeitet werden können und die Arbeitslosigkeit in diesem Bereich chronisch ist. In anderen Bereichen, z. B. der Autoindustrie, wurden etwa 150 verschiedene Personenwagentypen zusammengesetzt, kapitalintensive Investitionen getätigt, die nur minimalen Zahlen von Personen einen Arbeitsplatz boten.

In dieser Situation übernehmen die Militärs im Oktober 1968 die Macht. Ihr Putsch ist unblutig, niemand denkt daran, die vorhergehende Regierung zu verteidigen. In der Folge versuchen die Militärs, die vorgeben, eine Revolution eigenen Stils zu machen, das ökonomische Chaos zu ordnen, die Industrialisierung zu beschleunigen und die unterschiedlichen Klassen und Sek toren der Gesellschaft miteinande $\mathrm{zu}$,versöhnen". Die Industrialisierung soll durch eine Kontrolle des Kapitalabflusses, Verstaatlichung der Banken, Verstaatlichung der Erdölraffinerien, Schutzzölle für die einheimische Industrie und Integration in den Andenpakt (gemeinsamer Markt Venezuelas, Kolumbiens, Ecuadors, Perus, Boliviens und Chiles) staatliche Planung und Lenkung durch Steuerpräferenzen gefördert werden. Eine Agrarreform soll den 
Industrialisierungsprozeß durch zweierlei Maßnahmen unterstützen. Einerseits sollen die vorherigen $G$ roßgrundbesitzer in den industriellen Sektor ubergeleitet werden. Die Entschädigungen, die sie für das (nachweislich zu irgendeinem Zeitpunkt von den indianischen Bauern geraubte) enteignete Land erhalten, sind durch Investitionen in der Industrie auslösbar, die begünstigten Tagelöhner oder feudal abhängigen Landarbeiter sollen die hierfür notwendigen Geldmittel als ,Agrarschuld", als Bezahlung für das ihnen zurückgegebene Land, über zwanzig Jahre hinweg able isten. Andererseits soll auf dem Land durch Schaffung einer breiten Schicht von Mittelbauern ein Markt für die Leichtindustrie des Landes aufgebaut werden. Durch eine Industriegesetzgebung, die den Arbeitern beschränkte Mitbestimmungsrechte und eine, wenn auch geringe, Kapitalbeteiligung sichern soll, will man die Klassen miteinander versöhnen, Arbeitskämpfe, insbesondere Klassenkämpfe, vermeiden. Gleichzeitig fördert man im ganzen Land gremiale Organisationen oder zumindest regierungskonforme Arbeiterorganisationen, die die vorher bestehenden Klassenorganisationen ersetzen sollen.

Eine Reihe der getroffenen Maßnahmen: Devisenkontrolle, Verstaatlichung des Außenhandels, Beteiligung der Arbeiter am Betriebskapital etc. werden vom nationalen Bürgertum als ,Revolution“ mißverstanden und die Maßnahmen, die es eigentlich fördern sollten, schlagen ins Gegenteil um. Die prixate Investitionstätigkeit stagniert weitgehend. Die Regierung verschafft sich ein antiimperialistisches Image in der Außenpolitik, welches einerseits die akademische Linke im Innern, andererseits die sozialistischen Länder für das Regime einnehmen soll. Staatliche Kontrollen und Lenkung fur eine Reihe der an das vorherige Konzessionssystem gewöhnten nordamerikanischen Konzerne führen zu einer Verstärkung dieses Bildes und zu einem Ruckgang der nordamerikanischen Investitionen. Das nordamerikanische Kapital wird in vielen Bereichen (Autoindustrie, Textilindustrie, Bergwerke) durch westeuropäisches bzw. japanisches Kapital ersetzt. Da kein nennenswerter anderer Weg zur Industrialisierung wirklich eingeschlagen wird, setzt das Regime weiterhin auf Kapitalinvestitionen aus dem Ausland. Die Militärs bieten ausländischen Kapitalgebern günstigste Konditionen und versuchen das Land (z. B. in Anzeigen in der New York Times) durch Sicherheit und Arbeitsfrieden für Kapitalgeber interessant darzustellen. Arbeitskämpfe müssen deshalb radikal unterdrückt und verschwiegen werden.

Trotzdem kommt es ab 1972 immer häufiger zu Arbeitskämpfen und Streiks, die in ihren Intentionen weit über das, Revolutionsmodell' der Militärs hinausgehen, auf die Verstaatlichung oder Arbeiterkontrolle von Betrieben abzielen. Aber nicht nur diese, sondern auch einfache Lohnkämpfe werden teils blutig unterdriickt, verheimlicht oder abgeschwächt. Gewerkschaftsführer werden verfolgt, der gewerkschaftliche Bauernführer Hugo Blanco z. B. deportiert.

Ein Teil der Leugnung von sozialen Konflikten drückt sich auch in den offiziösen Verlautbarungen und Erklärungen über die Ereignisse in Chile aus, die teils wörtlich lügnerische Behauptungen der chilenischen Militärs übernehmen.

Es ist kein Zufall, daß Publikationsorgane kritischer, in Opposition zur Regierung stehender Gruppen und Sozialwissenschaftler verboten werden müssen, wenn sie das Bild der friedlichen, alle Klassen versöhnenden ,Revolution' als Trugbild 
entlarven. Die Beschlagnahme der Zeitschrift ,Sociedad y Politica' und die Verfolgung ihrer Herausgeber ist kein Fehltritt der Regierung, obwohl sie im Gegensatz zu der von derselben Regierung erlassenen Pressegesetzgebung steht. Ihr Grund liegt in der Thematik des beschlagnahmten Heftes: einer Erklärung zum chilenischen Militärputsch und Materialien über die Politik der Militärregierung, die die zunehmende Unterdrückung von Arbeitern und Arbeitskämpfen denunzieren.

Sozialwissenschaftliche Veröffentlichungen sind nur solange genehm, wie sie der Regierung dienen. Peru ist noch nicht Chile. Die peruanischen Aibeiter haben nicht die kämpferische Tradition der chilenischen Arbeiter, das Bürgertum ist insgesamt schwächer als das chilenische. Es besteht die Gefahr, dabs das chilenische Beispiel und die allgemeine Brasilisierung Lateinamerikas die peruanischen Militärs zu einer noch härteren Verfolgung ihres Kurses bringen könnte. Solidarität mit den Arbeitern Perus und den Wissenschaftlern, die die Rechte der Ausgebeuteten verteidigen, ist notwendig.

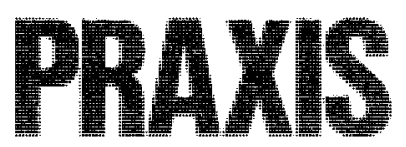

PHILOSOPHICAL QUARTERLY - INTERNATIONAL EDITION EDITORS: VELJKO KORAĆ AND GAJO PETROVIĆ

Vol. 9, No. 1, January-March 1973

\section{LIBERALISM AND SOCIALISM}

Mihailo Marković

Ljubomir Tadić

Trivo Indjic

Dobrica Cosić

Zdravko Kučinar

R. Bojanović, K. $\breve{C} a v o s ̌ k i, D . G r l i \hat{c}$,

B. Jakšić, D. Mičunović, Z. Golubović,

N. Rot, M. Zivotic
Opening of the Meeting

The Limits Set to Human Freedom by Private Property

Political Intervention and the Abstract Citizen

A Critique of Ideological a prion and Doctrinaire Attitudes

Some Observations Conceming

Liberalism and Marxism

Discussion

Annual subscription for 1973: 7.00

Annual subscription for 1974: Individuals $\$ 9.00$ (or DM 21,00)

Institutions $\$ 12.00$ (or DM 28,00)

Subscriptions are payable by cheque sent to:

PRAXIS, Filozof ski fakultet, 41000 Zagreb, Djure Salaja 3, Yugoslavia. 